\title{
ATMOSPHERIC PHASE DELAY IN SENTINEL SAR INTERFEROMETRY
}

\author{
Vrinda Krishnakumar ${ }^{1}$, Oriol Monserrat ${ }^{1}$, Michele Crosetto ${ }^{1}$, Bruno Crippa ${ }^{2}$ \\ ${ }^{1}$ Geomatics Division - Centre Tecnòlogic de Telecomunicacions de Catalunya (CTTC/CERCA) Av. Gauss, 7,Castelldefels \\ (Barcelona), Spain - (vrinda.krishnakumar,omonserrat,mcrosetto)@ cttc.cat \\ ${ }^{2}$ Department of Earth Sciences, Section of Geophysics, University of Milan, Via Cicognara 7, I-20129, Milan, Italy- \\ bruno.crippa@unimi.it
}

Commission III, WG III/3

KEY WORDS: Interferometric SAR, differential interferometry, tropospheric path delay

\begin{abstract}
:
The repeat-pass Synthetic Aperture Radio Detection and Ranging (RADAR) Interferometry (InSAR) has been a widely used geodetic technique for observing the Earth's surface, especially for mapping the Earth's topography and deformations. However, InSAR measurements are prone to atmospheric errors. RADAR waves traverse the Earth's atmosphere twice and experience a delay due to atmospheric refraction. The two major layers of the atmosphere (troposphere and ionosphere) are mainly responsible for this delay in the propagating RADAR wave. Previous studies have shown that water vapour and clouds present in the troposphere and the Total Electron Content (TEC) of the ionosphere are responsible for the additional path delay in the RADAR wave. The tropospheric refractivity is mainly dependent on pressure, temperature and partial pressure of water vapour. The tropospheric refractivity leads to an increase in the observed range. These induced propagation delays affect the quality of phase measurement and introduce errors in the topography and deformation fields. The effect of this delay was studied on a differential interferogram (DInSAR). To calculate the amount of tropospheric delay occurred, the meteorological data collected from the Spanish Agencia Estatal de Meteorología (AEMET) and MODIS were used. The interferograms generated from Sentinel-1 carrying C-band Synthetic Aperture RADAR Single Look Complex (SLC) images acquired on the study area are used. The study area consists of different types of scatterers exhibiting different coherence. The existing Saastamoinen model was used to perform a quantitative evaluation of the phase changes caused by pressure, temperature and humidity of the troposphere during the study. Unless the phase values due to atmospheric disturbances are not corrected, it is difficult to obtain accurate measurements. Thus, the atmospheric error correction is essential for all practical applications of DInSAR to avoid inaccurate height and deformation measurements.
\end{abstract}

\section{INTRODUCTION}

\subsection{Atmospheric Effects on InSAR}

Synthetic Aperture RADAR (SAR) is an extensive tool to measure the topography of the surface, its changes over time and also other changes in the surface (Rosen et al., 2000). In SAR interferometry, the deformation signal obtained from the Earth surface is mixed with topographic signal (Hanssen, 2001). To overcome this problem, differential interferogram is used. Differential Synthetic Aperture RADAR Interferometry or DInSAR is used in remote sensing for measuring Earth surface deformation (Doin et al., 2009). This technique is considered more accurate than InSAR as it is capable of providing relative measures up to few centimetres or less (Danklmayer et al., 2009). When an interferogram is formed from two SAR images, and a Digital elevation Model (DEM) to simulate and remove phase changes due to topography, a new phase image is formed (Gabriel et al., 1989). The final phase of the DInSAR consists of surface change phase contributions, atmospheric contribution to phase differences cannot be easily distinguished from surface displacements and thus has an impact on altitude and surface deformation measurements (Ferretti et al., 2007).

When a microwave signal propagates through the atmosphere, two types of errors may potentially be introduced viz. bending and propagation delays. The bending effect is negligible based on the incidence angles of the satellites (Boncori et al., 2006). Hence, in this study, we focus on the propagation delay caused by the atmosphere which is one of the main limitations of DInSAR
One of the most intractable problems with InSAR is the effect of the refractive atmosphere on the phase of the interferogram. Accuracy in determining the height and displacement is known to be affected by atmospheric propagation. Great efforts have been made to understand the properties of the atmospheric effects on InSAR and to develop methods to mitigate the same. Also the influence of atmosphere can also be a contributing factor for coherence loss (or lack of Persistent Scatterers-PS) and the need to deal with phase ambiguities or wrapped phases (Crosetto et al., 2011)

\subsection{Tropospheric Effects}

The atmosphere is divided into two major layers, ionosphere and troposphere (Boncori et al., 2009). The difference in the refractive indices of the atmospheric layers affect the propagation of electromagnetic waves. The repeat-pass InSAR shows random variations in phase due to atmospheric heterogeneities giving inaccurate measurements (Askne et al., 1987). Since the effect in path-length changes due to ionosphere is significantly small in X-band and C-band radar (Brcic et al., 2011), only tropospheric path delay is considered in this work.

We assume that signals propagate at known constant velocity to convert time delays and phase shifts to distance (Zebker et al., 1997). When the RADAR signals propagate through the Earth's atmosphere, the velocity is lowered leading to variable delays which affect the observations. The troposphere which is the lowest portion of the Earth's atmosphere contains $99 \%$ of water vapour and aerosols. The path delay due to troposphere is caused due to air refractivity gradients (Doin et al., 2009). The air refractivity gradients in the troposphere are due to the dry air 
pressure, temperature, air moisture and condensed water in clouds or rain.

The path delay is calculated using recorded weather data from AEMET and Moderate Resolution Imaging Spectroradiometer (MODIS) remote sensing data.

\section{MATERIALS AND METHODS}

\subsection{Materials and Methodology}

Aim of this work is to calculate the tropospheric delay in the phase of a three-pass DInSAR. The two processes that are generation of DInSAR and calculation of tropospheric path delay require two different data sets. DInSAR is generated using three Sentinel-1A SLC images acquired on the same site. The path delay is calculated using the ground level meteorological data obtained from the Spanish Agencia Estatal de Meteorología (AEMET) and also Moderate Resolution Imaging Spectroradiometer (MODIS) data acquired over the Canary islands lying within the latitude $(30,25)$ and longitude $(-20,-10)$. Details of the datasets are given in Table (1)

\begin{tabular}{|c|c|c|}
\hline \multicolumn{3}{|c|}{$\begin{array}{l}\text { Sentinel-1A Single-look complex images at Interferometric } \\
\text { wide-swath mode at } 250 \mathrm{~km} \text { and } 5 \times 20 \mathrm{~m} \text { spatial resolution. }\end{array}$} \\
\hline Seriel no & 1 (Master image) & 2 (Slave image) \\
\hline Sensor & $\begin{array}{l}\text { Sentinel-1 C-band } \\
\text { synthetic aperture } \\
\text { radar (SAR) at } \\
5.405 \mathrm{GHz}\end{array}$ & $\begin{array}{l}\text { Sentinel-1 C-band } \\
\text { synthetic aperture } \\
\text { radar (SAR) at } \\
5.405 \mathrm{GHz}\end{array}$ \\
\hline $\begin{array}{l}\text { Start Date } \\
\text { (yyyy/mm/dd) }\end{array}$ & 2014-11-05 & 2014-11-17 \\
\hline Orbit No. & 3143 & 3318 \\
\hline Area of study & \multicolumn{2}{|c|}{$\begin{array}{l}\text { Canary } \text { Islands-latitude }(30,25) \text {, } \\
\text { longitude }(-20,-10)\end{array}$} \\
\hline \multicolumn{3}{|c|}{ Meteorological data } \\
\hline 1. & \multicolumn{2}{|c|}{$\begin{array}{l}\text { Agencia Estatal de Meteorología, } \\
\text { AEMET,Spain }\end{array}$} \\
\hline 2. & \multicolumn{2}{|c|}{ MODIS cloud product Terra (MOD06) } \\
\hline
\end{tabular}

Table 1. Main parameters of input data.

The approach adopted in this work is as shown in the Figure (1)

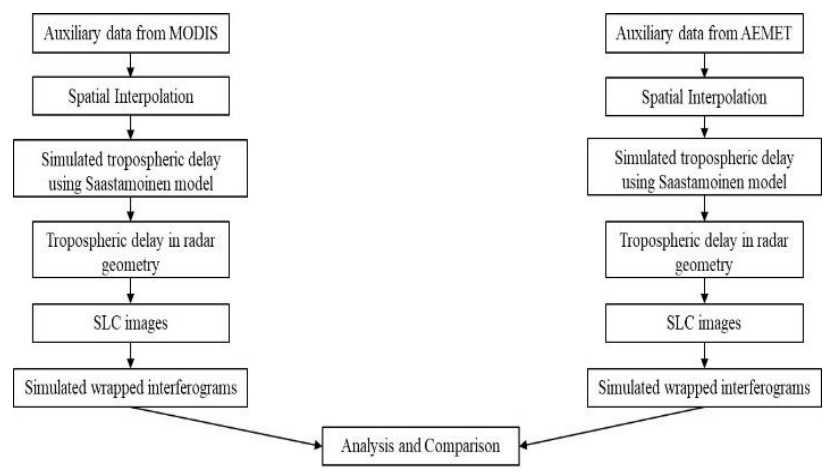

Figure 1. Flowchart for the methodology.

\subsection{Saastamoinen Model}

A simplified form of the Saastamoinen model used by Hanssen and Feijt (2011) was utilized in this work to make a first quantitative evaluation of tropospheric effects on InSAR measurements. When a signal propagates through a medium with refractive index unequal to 1 , an incremental path length due to the signal delay in the medium will happen.

Through various experiments, the incremental path length can be approximated by integrating the parameters (atmospheric pressure, temperature and partial pressure of water vapour) over the total path length in the troposphere. The incremental path length $\Delta \mathrm{R}_{\mathrm{e}}(\mathrm{m})$ in the model is expressed as a function of pressure, relative humidity, temperature and inclination as given below.

$$
\Delta \mathrm{R}_{\mathrm{e}}=2 * 10^{-6} \int_{0}^{\mathrm{H}} \frac{\mathrm{N}}{\cos \theta} \mathrm{dh}
$$

Where,

$\mathrm{H}=$ Vertical distance travelled by the RADAR wave in $\mathrm{m}$

$\mathrm{N}=$ Refractivity

$\theta=$ Incidence angle

$\mathrm{H}=$ Topographic height

The tropospheric refractivity depends on the pressure, temperature and partial pressure of water vapour. The formula used to calculate refractivity as given in

$$
\mathrm{N}=\left(77.6 \frac{\mathrm{P}}{\mathrm{T}}\right)-\left(5.6 \frac{\mathrm{e}}{\mathrm{T}}\right)-\left(0.375 * 10^{-6} \frac{\mathrm{e}}{\mathrm{T}^{2}}\right)
$$

Where,

$\mathrm{P}=$ Atmospheric pressure in $\mathrm{hPa}$

$\mathrm{T}=$ Temperature in Kelvin

$\mathrm{e}=$ Partial pressure of water vapour in $\mathrm{hPa}$

The partial pressure of water vapour can be derived from relative humidity as given below (Buck, 1981)

$$
\mathrm{e}=0.01 \exp \left(\begin{array}{c}
-2991.2729 \mathrm{~T}^{-2}-6017.0 .128 \mathrm{~T}^{-1}+18.87643854-0.028354721 \mathrm{~T} \\
+0.1788301 * 10^{-4} \mathrm{~T}^{-2}-0.84150417^{*} 10^{-9} \mathrm{~T}^{3} \\
+0.44412543^{*} 10^{-12} \mathrm{~T}^{4}+2.858487 \ln \mathrm{T}
\end{array}\right)
$$

The interferogram on which the tropospheric path delay has been calculated are shown in Figure (2). Since these interferograms exhibited characteristic presence of atmospheric turbulence by qualitative examination, the corresponding day's tropospheric phase delays were simulated using the data available.

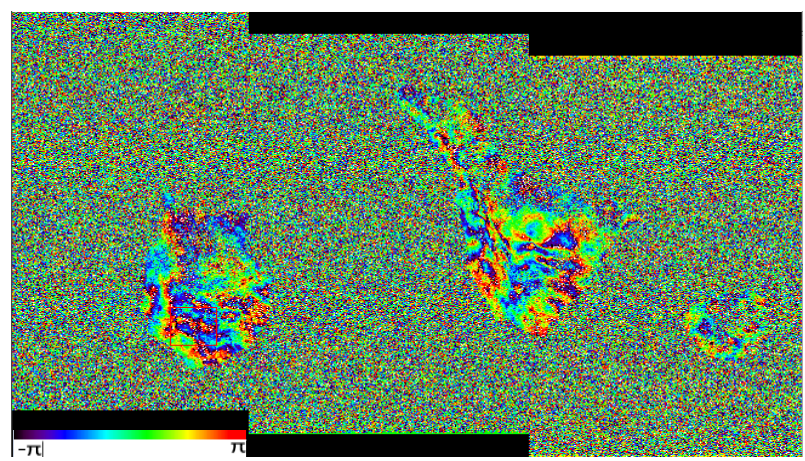

Figure 2. Interferogram with atmospheric artefacts generated from Sentinel master and slave

\section{RESULTS AND DISCUSSION}

\subsection{Tropospheric path delay using station data}

The data from only 15 weather stations of AEMET situated in and around Canary Islands were available for the selected dates. The point data was then used to build the model and later interpolated at the resolution of the DEM used for topographic phase removal. Initial tropospheric path delays obtained for the 
two days for which the interferogram was formed is given in Figure (3). Because of low spatial sampling of the data especially in the two smaller islands of La Gomera and Gran Canaria, no substantial difference in the path delay is observed. But a simple qualitative examination of the generated delay indicates an inflation in the tropospheric path delay towards the coast.

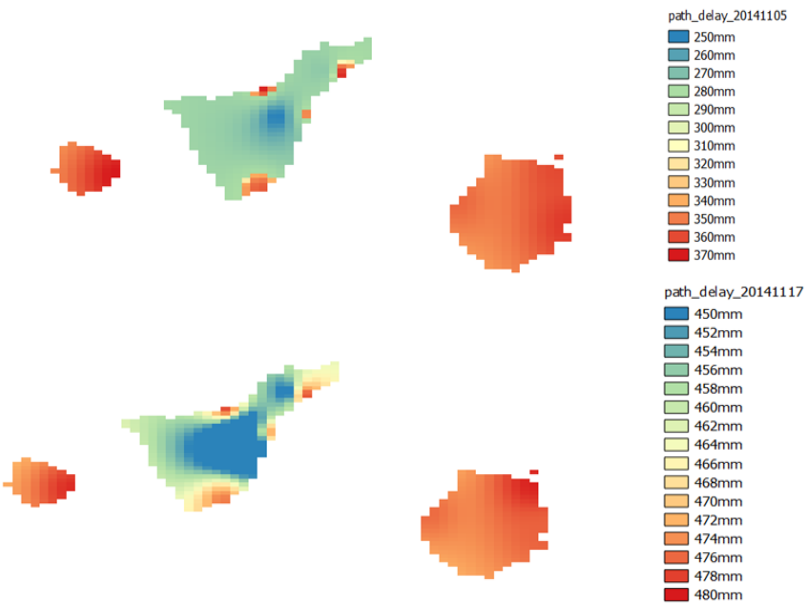

Figure 3. Master and slave tropospheric path delays from AEMET interpolated station data

The path elongation caused due to the refractive index of atmosphere introduces errors in the phase of an interferogram. For a differential interferogram, these variations in the phase can introduce considerable impact on the computations performed. The phase of the interferogram formed from the tropospheric delay of Master Image and Slave image 1 (Pair 1) is given in Figure (4).

Poor sampling of the data has resulted in a pattern different from the one in the original interferogram. Hence it is difficult to use this simulated tropospheric phase delay for further interpretations and calculations.

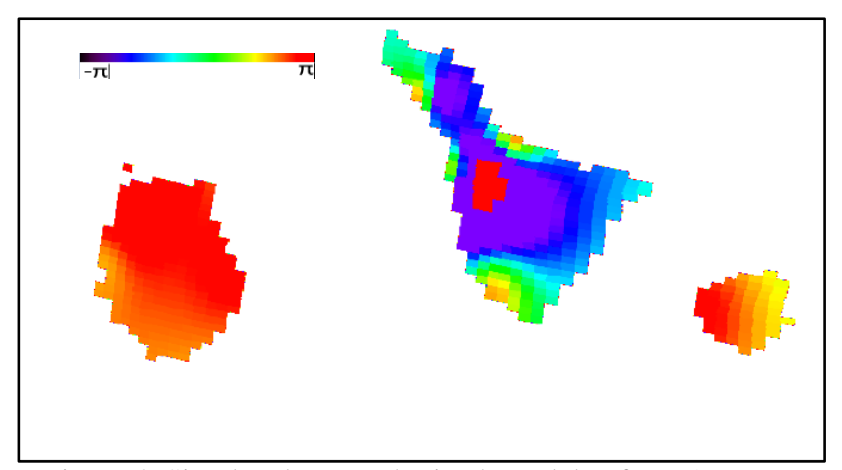

Figure 4. Simulated tropospheric phase delay from AEMET interpolated station data in RADAR geometry

\subsection{Tropospheric path delay using MODIS data}

In the next step the HDF formatted MODIS files once downloaded were re-projected to the ground geometry of the study area. The temperature and pressure bands required for path delay calculation from all the two images were extracted from MODIS cloud product MOD06. The tropospheric path delay calculate from MODIS data is shown in Figure (5).
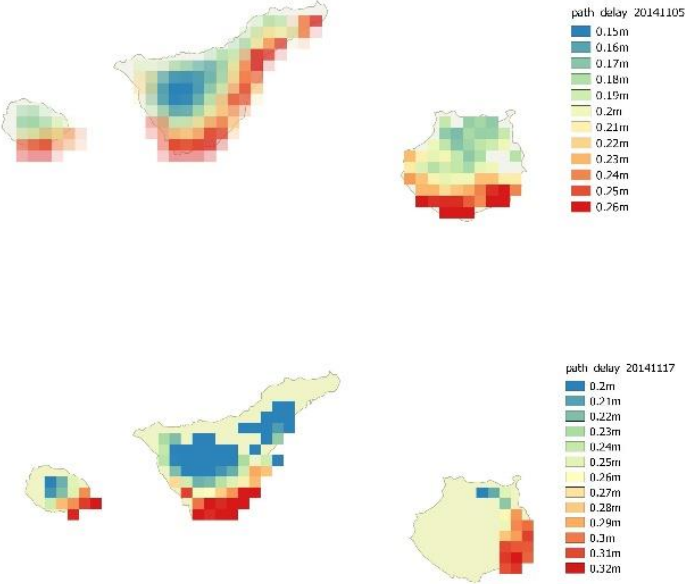

Figure 5. Master and Slave tropospheric path delays from MODIS data

The MODIS data acquired for Canary Islands had severe data gaps and had very low resolution. The availability of surface data at good sampling rate spatially and temporally was the main challenge faced while using the Saastamoinen model for tropospheric phase correction.

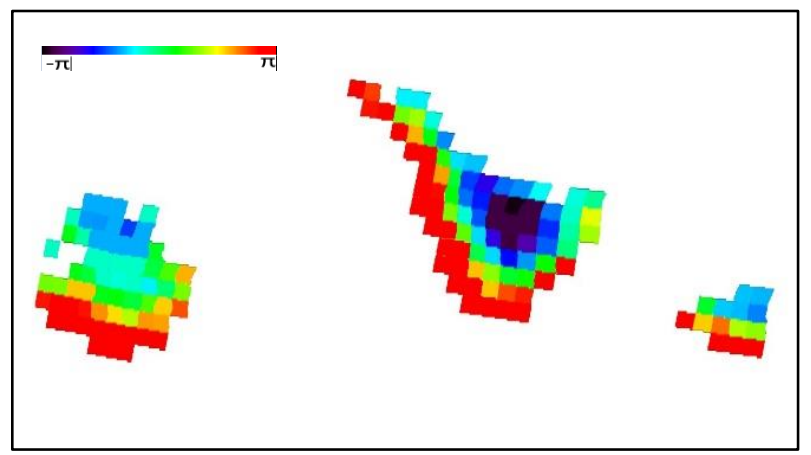

Figure 6. Simulated tropospheric phase delay from MODIS data in RADAR geometry

\section{CONCLUSION}

When the ground data gave a tropospheric phase delay of $0.25 \mathrm{~m}$ to $0.48 \mathrm{~m}$, the MODIS data resulted in a delay of $0.15 \mathrm{~m}$ to 0.32 $\mathrm{m}$. Obviously, the tropospheric path delay calculated using MODIS data is poorly estimated compared to the one from the station data. The results can be improved if input data at a greater spatial density is available. Integrating GPS measurements to the model will help to arrive at the most effective atmospheric phase removal technique.

The phase of an interferogram is a combination of atmospheric phase, phase due to baseline decorrelation, phase due to topography and other phase noise. The atmospheric component in the interferometric phase severely hinders the measurement when deriving the height or computing the displacement. Effect of this error in phase unwrapping and displacement measurements is beyond the scope of this work. 


\section{REFERENCES}

Askne, J. and Nordius, H., 1987. Estimation of tropospheric delay for microwaves from surface weather data. Radio Science, 22(03), pp.379-386.

Brcic, R., Parizzi, A., Eineder, M., Bamler, R. and Meyer, F., 2011, July. Ionospheric effects in SAR interferometry: An analysis and comparison of methods for their estimation. In Geoscience and Remote Sensing Symposium (IGARSS), 2011 IEEE International (pp. 1497-1500). IEEE.

Boncori, M. and Peter, J., 2009. Error Modelling for SAR interferometry and signal processing issues related to the use of an encoding SAR transponder. Ph.D.Thesis, University of Rome.

Buck, A.L., 1981. New equations for computing vapor pressure and enhancement factor. Journal of applied meteorology, 20(12), pp.1527-1532.

Crosetto, M., Monserrat, O., Cuevas, M. and Crippa, B., 2011. Spaceborne differential SAR interferometry: Data analysis tools for deformation measurement. Remote Sensing, 3(2), pp.305318.

Danklmayer, A., Doring, B.J., Schwerdt, M. and Chandra, M., 2009. Assessment of atmospheric propagation effects in SAR images. IEEE Transactions on Geoscience and Remote Sensing, 47(10), pp.3507-3518.

Doin, M.P., Lasserre, C., Peltzer, G., Cavalié, O. and Doubre, C., 2009. Corrections of stratified tropospheric delays in SAR interferometry: Validation with global atmospheric models. Journal of Applied Geophysics, 69(1), pp.35-50.

Ferretti, A., Monti Guarnieri, A., Prati, C., Rocca, F. and Massonnet, D., 2007. INSAR Principles B. ESA publications.

Gabriel, A.K., Goldstein, R.M. and Zebker, H.A., 1989. Mapping small elevation changes over large areas: differential radar interferometry. Journal of Geophysical Research: Solid Earth, 94(B7), pp.9183-9191.

Hanssen, R. and Feijt, A., 1997, March. A first quantitative evaluation of atmospheric effects on SAR interferometry. In ERS SAR Interferometry (Vol. 406, p. 277).

Hanssen, R.F., 2001. Radar interferometry: data interpretation and error analysis (Vol. 2). Springer Science \& Business Media.

Rosen, P.A., Hensley, S., Joughin, I.R., Li, F.K., Madsen, S.N., Rodriguez, E. and Goldstein, R.M., 2000. Synthetic aperture radar interferometry. Proceedings of the IEEE, 88(3), pp.333382.

Zebker, H.A., Rosen, P.A. and Hensley, S., 1997. Atmospheric effects in interferometric synthetic aperture radar surface deformation and topographic maps. Journal of Geophysical Research: Solid Earth, 102(B4), pp.7547-7563. 